\title{
Estrategias de aprendizaje y rendimiento académico en los estudiantes de Ingeniería Agrícola de la Universidad Nacional «Santiago Antúnez de Mayolo»
}

Learning strategies and academic achievement in Agricultural Engineering students at the «Santiago Antúnez de Mayolo» National University.

\author{
Pedro Colonia Cerna ${ }^{1}$ y Teófanes Mejía Anaya ${ }^{1}$
}

\section{RESUMEN}

El objetivo de esta investigación fue determinar la relación entre las estrategias de aprendizaje y rendimiento académico en los estudiantes del primer año de la Escuela Profesional de Ingeniería Agrícola, Facultad de Ciencias Agrarias, Universidad Nacional «Santiago Antúnez de Mayolo», Huaraz - 2012. La investigación fue descriptiva, correlacional, y transversal; no se hizo manipulación de las variables independientes, se determinó la relación que existe entre la variable independiente (estrategias de aprendizaje) y la variable dependiente (rendimiento académico), y los datos se tomaron en un momento dado; se trabajó con una muestra de 30 estudiantes del primer año de la promoción 2012. Para la recolección de información se validó la encuesta por el criterio de cinco expertos para relacionar las estrategias de aprendizaje y el rendimiento académico. Los resultados encontrados son como sigue: los estudiantes sólo utilizan parte de las estrategias cognitivas, metacognitivas y manejo de recursos. El nivel de rendimiento académico se encuentra dentro de la categoría regular. Al analizar las estrategias cognitivas, metacognitivas y el rendimiento académico se confirmó la hipótesis nula $\mathrm{H}_{0}$ con valores de $\mathrm{X}^{2} \mathrm{cal}=12.94 \mathrm{y} \mathrm{X}^{2} \mathrm{cal}=2.65$ respectivamente. En cambio, al analizar las estrategias, manejo de recursos y el rendimiento académico se confirmó la hipótesis $\mathrm{H}_{1} \mathrm{X}^{2} \mathrm{cal}=17.37$.

Palabras clave: estrategias de aprendizaje; rendimiento académico; ingeniería agrícola.

\section{ABSTRACT}

This research objective was to determine the relationship between learning strategies and academic achievement in the first year students of the Professional School of Agricultural Engineering, Faculty of Agricultural Sciences, National University «Santiago Antúnez de Mayolo», Huaraz - 2012. The study was not experimental, descriptive, correlational and cross; because there was not manipulation of the

1 Universidad Nacional «Santiago Antúnez de Mayolo». Huaraz, Perú. 
independent variables, the relationship between the independent variable (learning strategies) and the dependent variable (academic performance) was determined, and the data were taken at a given time; it was worked with a sample of 30 students of the 2012 promotion. For data collection, the survey was validated by the criterion of five experts to relate learning strategies and academic achievement. The results are as follows: students only uses part of cognitive, metacognitive and resource management. The level of achievement is within the regular category. In analyzing the cognitive, metacognitive and academic performance $\mathrm{H}_{0}$ the null hypothesis was confirmed by values and $\mathrm{X}^{2} \mathrm{cal}=12.94$ and $\mathrm{X}^{2} \mathrm{cal}=2.65$ respectively. Instead analyzing resource management strategies and academic performance $\mathrm{X}^{2} \mathrm{cal}=17.37 \mathrm{H}_{1}$ hypothesis was confirmed.

Keywords: learning strategies; academic achievement; agricultural engineering.

\section{ICHIKLLACHAW}

Kay yachay ashichaw uryayarquu maa imanawshi kay Waraschaw - 2012chaw, Hatun Yachay Wasi «Santiago Antúnez de Mayolopa», Escuela Profesional de Ingeniería Agrícolanchaw, Facultad de Ciencias Agrarias Yachatsikuychaw, huk kaq watachaw yachakuqkuna imanawpis yachakuyanqanwan yachayninkunatawan (kay ishkan kayan tumaqkuna) tinkushqanta rikaayaanaapaqmi. Kay yachay ashichaw tariyanqaatam willakuyaa, tsaymi kashqa maa imanawshi llapan watachaw ishkan tanqanakuyan. Ishkan tumaqkunatam yatapaayarquu. Tariyarquu ishkan tanqanakuyanqantam: imanawpis yachakuywan yachay, munayanqaa hunaqmi rikapaayaarquu; uryayarquu 2012 watachaw kima chunka yachakuqkuna universidaaman yaykamuqkunawanmi. Tsaypaqmi inkuwistata yachaqkunawan chanintsatsiyarqaa. Kaychawmi kaykunata tariyaamurquu: yachakuqkuna iñishiyan ichik Tukuy ruraykuna cognitivas, metacognitivas, recursuskunatam. Yachakuqkuna yachakuyanqan kaykan pullanllachawmi. Estrategias cognitivastawan yackakuqkunapa yachaynintawan tinkutsirmi tariyaquu mana kaq hipuutisis nishqanta, tsaymi kashqa: Mana kaq hipuutisis nula H0 kay waaliqniwan $\mathrm{de} \mathrm{X} 2 \mathrm{cal}=12.94$ y X2cal $=2.65$. imanaw ruraykuna rekursustawan yachakuqpa yachawninta tinkutsir tariyarquu hipuutisis H1 X2cal = 17.37.

Pushaq shimikuna: imanawpis yachakuy; yachakuqpa yachaynin; murukuy inginiiru.

\section{INTRODUCCIÓN}

Esta investigación determina la relación entre las estrategias de aprendizaje y rendimiento académico en los estudiantes del primer año de la Escuela de Ingeniería Agrícola, Facultad de Ciencias Agrarias de la Universidad Nacional «Santiago Antúnez de Mayolo» (UNASAM) - Huaraz, 2012.

En la actualidad, nos encontramos en una sociedad en que la ciencia y el conocimiento avanzan vertiginosamente, el mercado laboral y profesional se vuelve cada vez más selectivo y competitivo; es decir, se busca personas muy capacitadas para desempeñarse en las diversas áreas laborales con eficiencia y eficacia. Según la UNESCO (1996) la universidad es una institución que pertenece a la sociedad, a cuyas demandas y necesidades debe responder. 
En las Universidades de Latinoamérica como manifiesta Garbanzo (2007) en el marco de la globalización, la educación superior enfrenta la necesidad de una convergencia y articulación de sus sistemas, así como la construcción y consolidación, que son importantes desafíos ante la fragmentación y la diversificación institucional. Los problemas, en la educación superior de las universidades de América Latina se pueden señalar, como la escasez de docentes con postgrados, la desactualización de los currículos, el uso de metodologías tradicionales, las carencias de orientación vocacional (UNESCO, 2003).

En el Perú, según Thorne (2000) se ha identificado la presencia de aspectos críticos en la educación superior, como la disminución en la exigencia y criterios de selección de los postulantes en el ingreso a las universidades, la baja calificación académica del docente universitario, pues sólo el $47 \%$ de docentes consignan tener postgrado, insuficiente fomento de la investigación, que en promedio solo el $2.4 \%$ de investigaciones las realiza el profesor universitario, en un período de 5 años y así la falta de sistemas de acreditación institucional, lo hace imprescindible para el mejoramiento de la calidad.

Por su parte, Trahtemberg (2006) manifiesta que, en la conferencia anual de ejecutivos (44 CADE) concluyeron que los estudiantes peruanos tienden a la memorización, resuelven los problemas mecánicamente, no tienen hábitos de lectura, presentan poca capacidad crítica, limitándose a realizar lo que el profesor les indica. Lo mismo afirma Kohler (2008). El sistema universitario peruano, se caracteriza por serias deficiencias que se reflejan en el rendimiento académico. Estas deficiencias no se inician en la formación superior, sino devienen de la formación básica y se agudizan en la universidad.

El bajo rendimiento y el alto índice de desaprobados, por uso inadecuado de estrategias de aprendizaje es una realidad que ocurre en la Facultad de Ciencias Agrarias y al interior de otras Facultades de la UNASAM, así como también en el ámbito de la región. El problema de la investigación es determinar, cuál es la relación entre las estrategias de aprendizaje y rendimiento académico en los estudiantes del primer año de la Escuela de Ingeniería Agrícola, Facultad de Ciencias Agrarias, UNASAM - Huaraz, 2012.

El objetivo general de la investigación es determinar la relación entre las estrategias de aprendizaje y rendimiento académico en los estudiantes del primer año de la Escuela de Ingeniería Agrícola, Facultad de Ciencias Agrarias, UNASAM - Huaraz, 2012. El objetivo específico, es identificar estrategias de aprendizaje, nivel de rendimiento, determinar la relación entre las estrategias cognitivas, metacognitivas y manejo de recursos en los estudiantes del primer año de la Escuela de Ingeniería Agrícola, Facultad de Ciencias Agrarias - UNASAM - Huaraz, 2012.

En la hipótesis del estudio se formula alternativas como: Existe relación directa y significativa entre las estrategias de aprendizaje y rendimiento académico en los estudiantes del primer año de la Escuela de Ingeniería Agrícola, Facultad de Ciencias Agrarias UNASAM - Huaraz - 2012. Y no existe relación entre las estrategias de aprendizaje y rendimiento académico en los estudiantes del primer año de la Escuela de Ingeniería Agrícola, Facultad de Ciencias Agrarias, UNASAM - Huaraz, 2012. 


\section{MATERIALES Y MÉTODOS}

La investigación realizada corresponde a una metodología mixta, porque analiza la variable estrategias de aprendizaje en forma cualitativa y la variable rendimiento académico en forma cuantitativa. Como lo manifiesta Carrasco (2009) el diseño es correlacional propositivo no experimental de corte transversal. El tipo de estudio por su finalidad es investigación aplicada; por el carácter de medida es investigación mixta; por la profundidad, es descriptiva - correlacional, por el alcance temporal es transversal y por su orientación es de aplicación como lo señalan Hernández, Fernández y Batista, (2010); los instrumentos fueron cuestionarios de preguntas cerradas, conformados por 31 preguntas con tres posibilidades de respuesta en cada una y para determinar el rendimiento académico de los 30 alumnos que forman la muestra de estudio. Se solicitó a la OGE las actas de notas finales de las asignaturas de: Sociología General, Taller de Comunicación, Axiología y Deontología General, Matemática Básica, Química General, Biología General, Matemática I, Análisis Económico, Dibujo de Ingeniería I, Dibujo Técnico, Geología General y Meteorología; y el procesamiento de los datos se realizó a través del paquete estadístico SPSS versión 21.0 con un nivel de significancia con valor de $\mathrm{P}=0.05$.

\section{RESULTADOS}

En esta parte del trabajo se presenta las evidencias y los hallazgos, los cuales son mostrados en detalle en las tablas con los resultados correspondientes.

Tabla 1. Distribución numérica y porcentual de las dimensiones de las estrategias de aprendizaje

\begin{tabular}{lcc}
\hline $\begin{array}{c}\text { Dimensiones de las Estrategias } \\
\text { de Aprendizaje }\end{array}$ & Número & Porcentaje \\
\hline Estrategias Cognitivas & 19 & $63.3 \%$ \\
Bueno & 9 & $30.0 \%$ \\
Regular & 2 & $6.7 \%$ \\
Deficiente & & \\
Estrategias Metacognitivas & 26 & $86.7 \%$ \\
Bueno & 3 & $10.0 \%$ \\
Regular & 1 & $3.3 \%$ \\
Deficiente & & \\
Manejo de Recursos & 15 & $50.0 \%$ \\
Bueno & 13 & $43.3 \%$ \\
Regular & 2 & $6.7 \%$ \\
Deficiente & & \\
Estrategias de Aprendizaje & 8 & $26.7 \%$ \\
Bueno & 21 & $70.0 \%$ \\
Regular & 1 & $3.3 \%$ \\
Deficiente $\quad$ Total & $\mathbf{3 0}$ & $\mathbf{1 0 0 \%}$ \\
\hline
\end{tabular}


En la tabla 1 se observa que el 63.3\% obtiene nivel Bueno respecto a las estrategias cognitivas, el 30.0\% tiene nivel regular, y el 6.7\% el nivel deficiente; el 86.7\% tiene nivel Bueno respecto a las estrategias metacognitivas, el 10.0\% obtiene nivel regular y el $3.3 \%$ nivel deficiente; el 50.0\% alcanza nivel Bueno respecto al manejo de recursos; el $43.3 \%$, obtiene nivel regular y $6.7 \%$ el nivel deficiente.

Tabla 2. Distribución numérica y porcentual de las dimensiones del rendimiento académico

\begin{tabular}{|c|c|c|}
\hline $\begin{array}{c}\text { Dimensiones del Rendimiento } \\
\text { Académico }\end{array}$ & Número & Porcentaje \\
\hline \multicolumn{3}{|l|}{ Cultura General } \\
\hline Bueno & 1 & $3.4 \%$ \\
\hline Regular & 25 & $83.3 \%$ \\
\hline Deficiente & 4 & $13.3 \%$ \\
\hline Pésimo & 0 & $0.0 \%$ \\
\hline \multicolumn{3}{|l|}{ Formación Básica } \\
\hline Bueno & 0 & $0.0 \%$ \\
\hline Regular & 17 & $56.7 \%$ \\
\hline Deficiente & 12 & $40.0 \%$ \\
\hline Pésimo & 1 & $3.3 \%$ \\
\hline \multicolumn{3}{|l|}{ Cursos Propedéuticos } \\
\hline Bueno & 0 & $0.0 \%$ \\
\hline Regular & 14 & $46.7 \%$ \\
\hline Deficiente & 14 & $46.7 \%$ \\
\hline Pésimo & 2 & $6.6 \%$ \\
\hline \multicolumn{3}{|l|}{ Rendimiento Académico } \\
\hline Bueno & 00.00 & $0.0 \%$ \\
\hline Regular & 19 & $63.3 \%$ \\
\hline Deficiente & 10 & $33.3 \%$ \\
\hline Pésimo & 1 & $3.4 \%$ \\
\hline Total & 30 & $100 \%$ \\
\hline
\end{tabular}

En la tabla 2 se observa que el 3.4\% obtiene nivel bueno, el 83.3\% alcanza el nivel regular, el $13.3 \%$ nivel deficiente y el $0.0 \%$ nivel pésimo respecto a los cursos de cultura general; el $0.0 \%$ obtiene el nivel bueno, el 56.7\% nivel regular, el 40.0\% nivel deficiente y el 3.3\% nivel pésimo respecto a la formación básica; el $0.0 \%$ obtiene nivel bueno, el $46.7 \%$ obtiene nivel regular y deficiente, y el 6.6\% nivel pésimo respecto a los cursos propedéuticos y a nivel general el 63.3\% obtiene nivel regular, el 33.3\% nivel deficiente y el 3.4\% nivel pésimo respecto al rendimiento académico. 
Tabla 3. Distribución porcentual de las estrategias cognitivas y rendimiento académico

\begin{tabular}{|c|c|c|c|c|c|c|c|c|}
\hline \multirow{3}{*}{$\begin{array}{l}\text { ESTRATEGIAS } \\
\text { COGNITIVAS }\end{array}$} & \multicolumn{8}{|c|}{ RENDIMIENTO ACADÉMICO } \\
\hline & \multicolumn{2}{|c|}{ Regular } & \multicolumn{2}{|c|}{ Deficiente } & \multicolumn{2}{|c|}{ Pésimo } & \multicolumn{2}{|c|}{ Total } \\
\hline & $\mathrm{N}^{\circ}$ & $\%$ & $\mathrm{~N}^{\circ}$ & $\%$ & $\mathrm{~N}^{\circ}$ & $\%$ & $\mathrm{~N}^{\circ}$ & $\%$ \\
\hline Bueno & 8 & $26.7 \%$ & 10 & $33.3 \%$ & 1 & $3.3 \%$ & 19 & $63.3 \%$ \\
\hline Regular & 0 & $0.0 \%$ & 9 & $30.0 \%$ & 0 & $0.0 \%$ & 9 & $30.0 \%$ \\
\hline Deficiente & 2 & $6.7 \%$ & 0 & $0.0 \%$ & 0 & $0.0 \%$ & 2 & $6.7 \%$ \\
\hline Total & 10 & $33.4 \%$ & 19 & $63.3 \%$ & 1 & $3.3 \%$ & 30 & $100 \%$ \\
\hline $\mathrm{X}^{2} \mathrm{cal}=12.94$ & & & & $\begin{array}{l}\mathrm{X}^{2} \mathrm{tab}= \\
\mathrm{p}=0.88\end{array}$ & 4.067 & & $>0.05$ & \\
\hline
\end{tabular}

En la tabla 3 se observa que el $26.7 \%$ obtiene nivel bueno respecto a las estrategias cognitivas y nivel regular en el rendimiento académico; el $0.0 \%$ obtiene nivel regular respecto a las estrategias cognitivas y nivel regular en el rendimiento académico; en tanto que el $6.7 \%$ obtiene nivel deficiente respecto a las estrategias cognitivas y nivel regular en el rendimiento académico; así mismo, el 33.3\% alcanza nivel bueno respecto a las estrategias cognitivas y nivel deficiente en el rendimiento académico, el 30\% obtiene el nivel regular respecto a las estrategias cognitivas y deficiente en el rendimiento académico; además el 3.3\% obtiene el nivel de bueno respecto a las estrategias cognitivas y pésimo con respecto al rendimiento académico. También se observa que el valor de Chi-Cuadrado es $\mathrm{X}^{2} \mathrm{cal}=12.94<\mathrm{X}^{2} \mathrm{tab}=14.067$ con nivel de significancia $\mathrm{p}$ $=0.887$, al cual es mayor al 5\%. Se deduce que no existe relación significativa entre las estrategias cognitivas y el rendimiento académico.

Tabla 4. Distribución porcentual de las estrategias metacognitivas y rendimiento académico

\begin{tabular}{lcccccccc}
\hline \multicolumn{1}{c}{ ESTRATEGIAS } & \multicolumn{7}{c}{ RENDIMIENTO ACADÉMICO } \\
METACOGNITIVAS & \multicolumn{2}{c}{ Regular } & \multicolumn{2}{c}{ Deficiente } & \multicolumn{2}{c}{ Pésimo } & \multicolumn{2}{c}{ Total } \\
\hline & $\mathrm{N}^{\circ}$ & $\%$ & $\mathrm{~N}^{\circ}$ & $\%$ & $\mathrm{~N}^{\circ}$ & $\%$ & $\mathrm{~N}^{\circ}$ & $\%$ \\
Bueno & 15 & $50.0 \%$ & 10 & $33.3 \%$ & 1 & $3.3 \%$ & 26 & $86.7 \%$ \\
Regular & 3 & $10.0 \%$ & 0 & $0.0 \%$ & 0 & $0.0 \%$ & 3 & $10.0 \%$ \\
Deficiente & 1 & $3.3 \%$ & 0 & $0.0 \%$ & 0 & $0.0 \%$ & 1 & $3.3 \%$ \\
Total & 19 & $63.3 \%$ & 10 & $33.3 \%$ & 1 & $3.3 \%$ & 30 & $100 \%$ \\
$\mathrm{X}^{2}$ cal $=2.65$ & & & $\mathrm{X}^{2} \mathrm{tab}$ & $=14.067 \mathrm{p}=0.181$ & $>0.05$ & \\
\hline
\end{tabular}

En la tabla 4 se observa que el 50.0\% alcanza nivel bueno respecto a las estrategias metacognitivas y nivel regular en el rendimiento académico; el 10.0\% obtiene nivel regular respecto a las estrategias metacognitivas y tienen nivel regular en el rendimiento académico; el 3.3\% alcanza el nivel deficiente y regular en el rendimiento académico; en tanto que el 33.3\% llega al nivel bueno respecto a las estrategias metacognitivas y a la vez tiene nivel deficiente en el rendimiento académico; así mismo el 3.3\% obtiene nivel bueno respecto a las estrategias metacognitivas y nivel pésimo en el rendimiento académico. También se observa que el valor de Chi-Cuadrado es $\mathrm{X}^{2}$ cal $=2.65<$ $\mathrm{X}^{2} \mathrm{tab}=14.067$ con nivel de significancia $\mathrm{p}=0.181$, el cual es mayor al 5\%; por ende 
se deduce que no existe relación significativa entre las estrategias metacognitivas y el rendimiento académico.

Tabla 5. Distribución porcentual del manejo de recursos y rendimiento académico

\begin{tabular}{|c|c|c|c|c|c|c|c|c|}
\hline \multirow{3}{*}{$\begin{array}{l}\text { MANEJO DE } \\
\text { RECURSOS }\end{array}$} & \multicolumn{8}{|c|}{ RENDIMIENTO ACADÉMICO } \\
\hline & \multicolumn{2}{|c|}{ Regular } & \multicolumn{2}{|c|}{ Deficiente } & \multicolumn{2}{|c|}{ Pésimo } & \multicolumn{2}{|c|}{ Total } \\
\hline & $\mathrm{N}^{\circ}$ & $\%$ & $\mathrm{~N}^{\circ}$ & $\%$ & $\mathrm{~N}^{\circ}$ & $\%$ & $\mathrm{~N}^{\circ}$ & $\%$ \\
\hline Bueno & 4 & $13.3 \%$ & 10 & $33.3 \%$ & 1 & $3.3 \%$ & 15 & $50.0 \%$ \\
\hline Regular & 13 & $43.3 \%$ & 0 & $0.0 \%$ & 0 & $0.0 \%$ & 13 & $43.3 \%$ \\
\hline Deficiente & 2 & $6.7 \%$ & 0 & $0.0 \%$ & 0 & $0.0 \%$ & 2 & $6.7 \%$ \\
\hline Total & 19 & $\mathrm{~s} 63.3 \%$ & 10 & $33.3 \%$ & 1 & $3.3 \%$ & 30 & $100 \%$ \\
\hline $\mathrm{X}^{2} \mathrm{cal}=17.37$ & & & & $\mathrm{X}^{2} \mathrm{tab}=$ & 14.06 & & $\mathrm{P}=0.013$ & $<0.05$ \\
\hline
\end{tabular}

En la tabla 5 se observa que el 13.3\% alcanza nivel bueno respecto al manejo de recursos y a la vez, nivel regular en el rendimiento académico; el 43.3\% se ubica en el nivel regular respecto al manejo de recursos y alcanza nivel regular en el rendimiento académico; así mismo el 33.3\% obtiene nivel bueno respecto al manejo de recursos y a la vez nivel deficiente en el rendimiento académico. También se observa que el valor de Chi-Cuadrado es $\mathrm{X}^{2} \mathrm{cal}=17.37>\mathrm{X}^{2} \mathrm{tab}=14.067$ con nivel de significancia $\mathrm{p}=0.013$ el cual es menor al 5\%. Se deduce que existe relación significativa entre el manejo de recursos y el rendimiento académico.

\section{Contrastación de hipótesis}

Tabla 6. Análisis correlacional de las estrategias de aprendizaje y rendimiento académico

\begin{tabular}{|c|c|c|c|c|c|c|c|}
\hline \multirow{3}{*}{$\begin{array}{l}\text { ESTRATEGIAS DE } \\
\text { APRENDIZAJE }\end{array}$} & \multicolumn{7}{|c|}{ RENDIMIENTO ACADÉMICO } \\
\hline & \multicolumn{2}{|c|}{ Regular } & \multicolumn{2}{|c|}{ Deficiente } & \multicolumn{2}{|c|}{ Pésimo } & \multirow[t]{2}{*}{ TOTAL } \\
\hline & $\mathrm{O}$ & $\mathrm{E}$ & $\mathrm{O}$ & $\mathrm{E}$ & $\mathrm{O}$ & $\mathrm{E}$ & \\
\hline Bueno & 10 & 13.30 & 10.00 & 7.00 & 1.00 & 0.70 & 21 \\
\hline Regular & 8 & 5.07 & 0.00 & 2.67 & 0.00 & 0.27 & 8 \\
\hline Deficiente & 1 & 0.63 & 0.00 & 0.33 & 0.00 & 0.03 & 1 \\
\hline TOTAL & \multicolumn{2}{|c|}{19} & \multicolumn{2}{|c|}{10} & \multicolumn{2}{|c|}{1} & 30 \\
\hline
\end{tabular}

Leyenda: O: Valores Observados E: Valores Esperados

Cálculo de Chi-Cuadrado:

$$
\begin{gathered}
X^{2}=\frac{\sum(O-E)^{2}}{E}=\frac{(10-13.30)^{2}}{13.30}+\frac{(8-5.07)^{2}}{5.07}+\frac{(1-0.63)^{2}}{0.63}+\frac{(10-7)^{2}}{7}+\frac{(0-2.67)^{2}}{2.67}+\frac{(0-0.33)^{2}}{0.33} \\
\frac{(1-0.7)^{2}}{0.7}+\frac{(0-0.27)^{2}}{0.27}+\frac{(0-0.03)^{2}}{0.03}=7.44
\end{gathered}
$$


Región crítica:

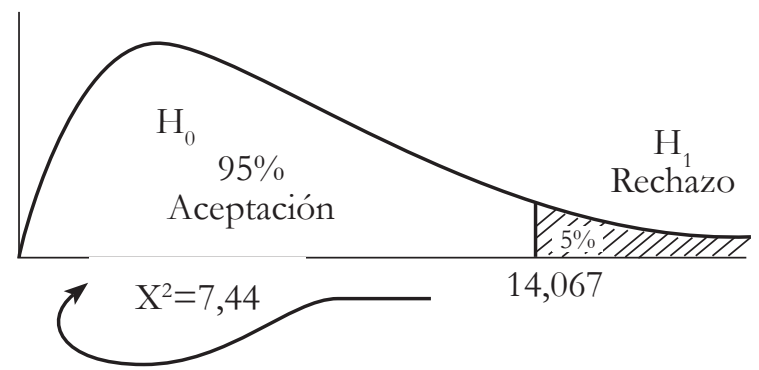

En la tabla 6 del análisis correlacional se observa, que en la región no crítica el valor de Chi-cuadrado es 7.44, el cual cae en la región de aceptación de la hipótesis nula; es decir, se demuestra que no existe relación entre las estrategias de aprendizaje y el rendimiento académico.

\section{DISCUSIÓN}

Analizando los resultados en la tabla 1, en requerimiento del objetivo 1, para identificar las estrategias más empleadas se observa que el $63.3 \%$ obtiene nivel bueno respecto a las estrategias cognitivas; el 30.0\%, nivel regular, y el 6.7\% el nivel deficiente; el $86.7 \%$ alcanza el nivel bueno respecto a las estrategias metacognitivas; el 10.0\% obtiene nivel regular y el 3.3\% nivel deficiente; el 50.0\% tiene nivel bueno respecto al manejo de recursos el $43.3 \%$ alcanza nivel regular y $6.7 \%$ obtiene nivel deficiente.

Analizando los resultados de la tabla 2, en requerimiento del objetivo específico 2 para identificar el nivel del rendimiento académico se observa que el $3.4 \%$ obtiene nivel bueno, el $83.3 \%$ el nivel regular, el 13.3\% nivel deficiente y el $0.0 \%$ obtiene nivel pésimo respecto a los cursos de cultura general; el $0.0 \%$ no llega a nivel bueno, el $56.7 \%$ nivel regular, el $40.0 \%$ nivel deficiente y el 3.3\% nivel pésimo respecto a la formación básica; el $0.0 \%$ no llega al nivel bueno, el 46.7\% alcanza nivel regular y deficiente, y el 6.6\% nivel pésimo respecto a los cursos propedéuticos y, a nivel general, el 63.3\% alcaza nivel regular, el 33.3\% nivel deficiente y el 3.4\% nivel pésimo respecto al rendimiento académico de los estudiantes del primer año de Ingeniería Agrícola de la Facultad de Ciencias Agrarias. Además se observa que de los 30 alumnos, el promedio global anual es de 11.1 puntos que, según la escala vigesimal, se encuentra dentro de la categoría regular.

Analizando los resultados de la tabla 3, según requerimiento del objetivo específico 3, para determinar la relación entre las estrategias cognitivas y rendimiento académico, se observa que el $26.7 \%$ obtiene nivel bueno respecto a las estrategias cognitivas y a la vez tiene nivel regular en el rendimiento académico; el 0.0\% obtiene nivel regular respecto a las estrategias cognitivas y nivel regular en el rendimiento académico; en tanto que el $6.7 \%$ obtiene nivel deficiente respecto a las estrategias cognitivas y a la vez, nivel regular en el rendimiento académico; así mismo, el 33.3\% alcanza nivel bueno respecto a las estrategias cognitivas y a la vez, nivel deficiente en el rendimiento académico; el $30 \%$ obtiene el nivel regular respecto a las estrategias cognitivas y a la vez, deficiente en el rendimiento académico; además el 3.3\% obtiene el nivel bueno respecto a las estrategias cognitivas y pésimo con respecto al rendimiento académico. También se observa que el valor de Chi-Cuadrado es $\mathrm{X}^{2} \mathrm{cal}=12.94<\mathrm{X}^{2} \mathrm{tab}=14.067$ con nivel de significancia $\mathrm{p}=0.887$, el cual es mayor al $5 \%$, por ende se deduce que no existe relación significativa entre las estrategias cognitivas y el rendimiento académico. 
Analizando los resultados de la tabla 4 en armonía con el objetivo específico 4, para determinar la relación entre las estrategias metacognitivas y rendimiento académico se observa que el $50.0 \%$ obtiene nivel bueno respecto a las estrategias matacognitivas y a la vez, nivel regular en el rendimiento académico; el 10.0\% obtiene nivel regular respecto a las estrategias metacognitivas y nivel regular en el rendimiento académico, y el 3.3\% obtiene el nivel deficiente y regular en el rendimiento académico; en tanto que el 33.3\% alcanza nivel bueno respecto a las estrategias metacognitivas y a la vez nivel deficiente en el rendimiento académico; así mismo el 3.3\% logra nivel bueno respecto a las estrategias metacognitivas y a la vez, nivel pésimo en el rendimiento académico. También se observa que el valor de Chi-Cuadrado es $\mathrm{X}^{2} \mathrm{cal}=2.65<\mathrm{X}^{2}$ tab $=14.067$ con nivel de significancia $\mathrm{p}=0.181$, el cual es mayor al $5 \%$, por ende se deduce que no existe relación significativa entre las estrategias metacognitivas y el rendimiento académico.

Analizando los resultados de la tabla 5 en atención al requerimiento del objetivo específico 5, para determinar la relación entre las estrategias de manejo de recursos y rendimiento académico se observa que el 13.3\% alcanza nivel bueno respecto al manejo de recursos y a la vez, nivel regular en el rendimiento académico, el 43.3\% logra nivel regular respecto al manejo de recursos y nivel regular en el rendimiento académico; el $6.7 \%$ obtiene el nivel deficiente respecto al manejo de recursos y regular en el rendimiento académico; así mismo el 33.3\% alcanza nivel bueno respecto al manejo de recursos y a la vez, nivel deficiente en el rendimiento académico; el 3.3\% obtiene nivel bueno respecto al manejo de recursos y a la vez nivel pésimo en el rendimiento académico. También se observa que el valor de Chi-Cuadrado es $\mathrm{X}^{2}$ cal $=17.37>\mathrm{X}^{2}$ tab $=14.067$ con nivel de significancia $\mathrm{p}=0.013$, el cual es menor al $5 \%$, por ende se deduce que existe relación significativa entre el manejo de recursos y el rendimiento académico.

Examinando los resultados de la tabla 6 del análisis correlacional de las estrategias de aprendizaje y rendimiento académico se observa que, en la región no crítica el valor de Chi-cuadrado es 7.44, el cual cae en la región de aceptación de la hipótesis nula (Ho); es decir, se demuestra que no existe relación entre las estrategias de aprendizaje y el rendimiento académico en los estudiantes de primer año de la Escuela Profesional de Ingeniería Agrícola de la Facultad de Ciencias Agrarias de la UNASAM. Se determina que estos resultados son similares a los encontrados por Sotil y Quintana (2002), en la tesis de investigación «Influencias del clima familiar, estrategias de aprendizaje e inteligencia emocional en el rendimiento académico» que, evaluados a través del promedio ponderado de las calificaciones obtenidas por los estudiantes durante su permanencia en la universidad, no existe una correlación significativa.

Estos resultados también tienen relación con lo que afirma Torres (2002), en su tesis «Influencia de la motivación y las estrategias de aprendizaje en el rendimiento académico de estudiantes universitarios» según los resultados la motivación y las estrategias de aprendizaje están relacionadas con el rendimiento académico de los alumnos, entendiéndose por estrategias según Beltrán y Jesús (1988) las «medidas que sirven para mejorar la calidad del rendimiento de los estudiantes, que implica una toma de decisiones y un plan de acción».

Como parte de nuestra experiencia podemos afirmar que la calidad educativa es deficiente en la secundaria, poco o nada hacen uso de las estrategias de aprendizaje y cuando ingresan a la universidad no cuentan con el uso de estrategias de aprendizaje; para revertir esta situación se debe trabajar en forma individualizada con los alumnos o proponer un ciclo introductorio a la universidad. 


\section{CONCLUSIÓN}

Solo el 26.7\% emplea parte de las estrategias de aprendizaje investigadas. El nivel del rendimiento académico se encuentra al $63.3 \%$, con promedio de nota 11.1 , se ubica en la categoría regular. Pero sí existe relación significativa entre el manejo de recursos y el rendimiento académico. Del análisis correlacional se demuestra que no existe relación entre las estrategias de aprendizaje y el rendimiento académico.

\section{REFERENCIAS BIBLIOGRÁFICAS}

Beltrán, Luis y Álvaro, Jesús. 1998. Procesos, estrategias, técnicas de aprendiraje. 2da. reimpresión. Madrid: Síntesis.

Carrasco, Sergio. 2009. Metodología de la investigación cientifica. Lima: San Marcos.

Garbanzo, Guiselle. 2007. Factores asociados al rendimiento académico en estudiantes universitarios, una reflexión desde la calidad de la educación superior. Costa Rica: Universidad de Costa Rica, Vol. 3, N 1.

Hernández, Roberto; Fernández, Carlos y Batista, María del Pilar. 2010. Metodología de la investigación. $5^{\text {ta }}$ ed. México: Mc Graw-Hill.

Kohler, Johanna. 2008. Estrategias de aprendiraje y rendimiento académico de estudiantes de psicología del 1er al 4to ciclo de una universidad particular. Lima: Universidad de San Martín de Porres.

Sotil, Peña y Quintana, Brioso. 2002. «Influencias del clima familiar, estrategias de aprendizaje e inteligencia emocional en el rendimiento académico». Revista de investigación en Psicología, Vol.5, No 1, Lima.

Thahtemberg, León. 2006. «No saben Pensar». Lima.

Thorne, Cecilia. 2000. Indicadores de calidad de la universidad a nivel internacional y el caso peruano. Trabajo presentado en la reunión de especialistas en educación Universitaria. Lima: Consorcio de Universidades y Foro Educativo.

Torres, Valladares. 2002. Influencia de la Motivación y las estrategias de aprendizaje en el rendimiento académico de estudiantes universitarios. Lima: UNMSM.

UNESCO. 2003. El impacto del proceso de evaluación y acreditación en las universidades de América Latina. Caracas.

UNESCO. 1996. Documento sobre políticas para el cambio y el desarrollo de la educación superior. Caracas.

Fecha de recepción: 13 de noviembre 2015

Fecha de aceptación: 06 de diciembre 2015

\section{Correspondencia}

Pedro Colonia Cerna

Petercol29@hotmail.com 